\title{
DERIVAÇÃO VENTRICULAR E SUBDURAL BILATERAL COM A VÁLVULA DE HOLTER: NOVA TÉCNICA CIRÚRGICA
}

\author{
Walter C. Pereira * \\ Gilberto M. Almeida *
}

A ventriculo-auriculostomia com válvulas de fluxo unidirecional representa, atualmente, o tratamento de escolha da hidrocefalia $1,2,3,4,5,6,7$, $8,11,15,16$. Desde sua introdução na prática neurocirúrgica em 1952 por Nulsen e Spitz ${ }^{10}$ e em 1957 por Pudenz e col. ${ }^{13}$, numerosa casuística tem sido registrada na literatura, confirmando, cada vez mais, o seu valor.

Scarf 14 critica a ventrículo-auriculostomia, porém a maioria dos autores admite que êste método apresenta vantagens indiscutíveis sôbre as outras técnicas cirúrgicas empregadas para o tratamento da hidrocefalia, a despeito das complicações observadas em alguns casos ${ }^{2,9}$. Yashon ${ }^{16}$, em revisão de 147 casos de pacientes hidrocefálicos, 69 dos quais operados por diversas técnicas, chega à conclusão que êste tipo de derivação melhora o prognóstico desta moléstia, quanto à mortalidade, de 20 a $30 \%$. A maior vantagem da ventriculo-auriculostomia, no entanto, consiste na sua extrema simplicidade, podendo ser utilizada mesmo em casos graves, pràticamente sem mortalidade operatória.

Especialmente indicada em crianças com hidrocefalia comunicante tem sido, porém, realizada em pacientes com qualquer tipo de bloqueio ao trânsito do líquido cefalorraqueano (LCR) : como recurso paliativo em casos de tumores inoperáveis; para aliviar a hipertensão intracraniana em neoplasias que ulteriormente serão abordadas cirưrgicamente ou irradiadas e como tratamento definitivo de bloqueios inflamatórios do sistema ventricular ou do espaço subaracnóideo. Mais raramente tem sido empregada em casos de coleções subdurais, unilateralmente.

Vários trabalhos têm sido publicados relatando o emprêgo da ventrículoauriculostomia em hidrocefalias de causa tumoral 5, 6, 8. Assim, casos de neoplasias inoperáveis da fossa craniana posterior, da região do aqueduto cerebral ou da porção posterior do $3 .^{\circ}$ ventrículo, podem ser tratadas paliativamente através de "shunts" que desviam o fluxo do LRC bloqueado para a corrente venosa. Estas derivações são realizadas estabelecendo-se a comunicação de um dos ventrículos laterais com a aurícula direita, desde que haja permeabilidade dos orifícios interventriculares de Monro.

Trabalho da Clínica Neurológica da Faculdade de Medicina da Universidade de São Paulo (Prof. Adherbal Tolosa): * neurocirurgiões. 
Nas neoplasias da porção anterior do $3 .^{\circ}$ ventriculo, bem como da região supra-selar, pode ocorrer oclusão dos orifícios interventriculares, tornando necessário derivar o LRC de ambos os ventriculos laterais. As dificuldades técnicas para tais operaçōes são óbvias, sendo preciso recorrer a intervenções cirúrgicas trabalhosas, traumatisantes e de resultados inconstantes, como a operação de Torkildsen realizada bilateralmente ou a ventriculostomia transcalosa de Lazorthes, com abertura do septo pelúcido.

Idealizamos uma adaptação da ventrículo-auriculostomia para tais casos, derivando o LCR de ambos os ventrículos laterais por dois catéteres de borracha siliconizada conectados a uma sonda em $\mathbf{T}$ (sonda de Kehr) que, por sua vez, é posta em conexão com uma válvula de Holter (fig. 1). São praticadas duas trepanações na região parietal posterior, à cêrca de um centímetro do plano médio-sagital, segundo uma linha oblíqua, de tal maneira que a perfuração direita fique mais posterior que a esquerda; com isto conseguimos conectar a sonda de Kehr sem angulações que poderiam obstrui-la. Pelas perfurações são introduzidos nos ventriculos laterais dois catéteres de silicone, em forma de L, os quais são ligados, com conectores de polietileno, à sonda de Kehr; esta, por sua vez, é dirigida, sob o couro cabeludo, até a região retro-auricular onde é conectada à uma válvula de Holter. Os demais tempos da operação são idênticos aos da ventrículo-auriculostomia convencional 12,15 .

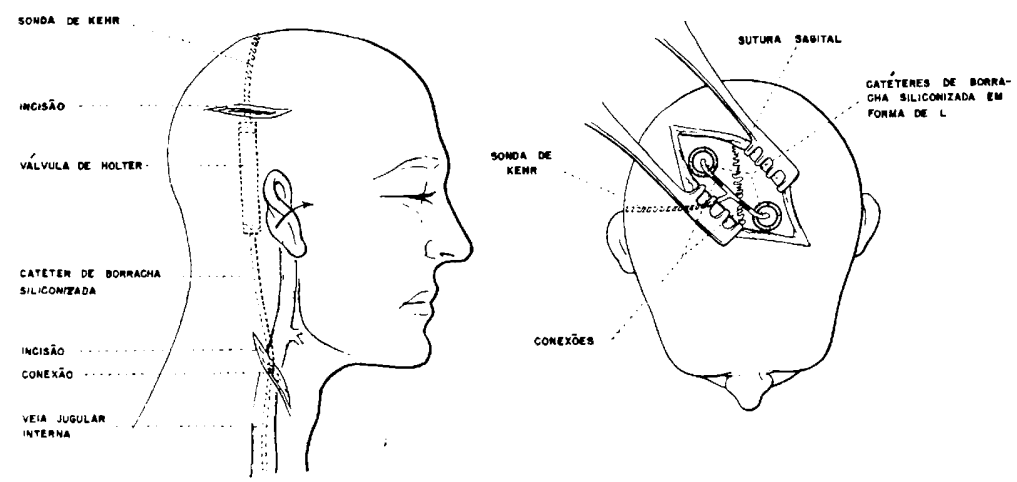

Fig. 1 - Esquema da técnica da derivação ventricular ou subdural bilateral, com a válvula de Holter. Explicações no texto.

Esta nova técnica pode ser também utilizada em casos de coleções subdurais bilaterais, derivando-se ambos os espaços subdurais para a aurícula direita. A vantagem dêste procedimento é evidente quando comparado às punções repetidas que, além de representarem risco constante de infecção, espoliam intensamente o paciente.

Até o presente empregamos a derivação bilateral em três casos: dois craniofaringiomas com bloqueio bilateral dos orificios interventriculares (A.P., 16 anos de idade, sexo masculino, côr branca, registro 743.864 e M.C.T., 11 anos de idade, sexo feminino, côr branca, registro 746.154) e uma coleção 
subdural bilateral, rebelde ao tratamento por punções repetidas (W.C.G., 6 meses de idade, sexo feminino, côr parda, registro 744.822).

Nos dois casos de craniofaringiomas houve regressão total do quadro de hipertensão intracraniana que ambos apresentavam em grau acentuado. $O$ caso A.P. teve alta após o desaparecimento do quadro hipertensivo, não tendo sido abordada a neoplasia por ser esta considerada inoperável; o caso M.C.T. pôde ser operado ulteriormente, em melhores condições gerais, decorrentes do alívio da intensa hipertensão intracraniana que agravava o sofrimento encefálico.

No caso de coleção subdural bilateral (W.C.G.), decorridos dois meses da derivação, foi realizada craniotomia esquerda para a retirada da cápsula, sendo verificada ausência de líquido em seu interior. Um mês depois foi feita craniotomia direita, constatando-se também total esgotamento da coleção, existindo apenas espaço virtual entre os folhetos da cápsula. Neste caso, durante algum tempo depois de instalada a derivação, a fontanela bregmática permaneceu tensa sendo necessário "bombar" a válvula freqưentemente. Éste fato deve ser atribuido à grande viscosidade do líquido da coleção, constituida de sangue hemolizado, que dificultava sua drenagem pelo sistema.

Apesar de pequeno o número de casos em que foi experimentada, a derivação bilateral com a válvula de Holter, parece-nos uma operação útil, sendo indicada em casos de bloqueio bilateral dos orificios de Monro e em coleções subdurais bilaterais, rebeldes ao tratamento por punções repetidas. Em ambos os casos seu emprêgo visa possibilitar melhores condições para intervenções definitivas ulteriores, ou tem finalidade exclusivamente paliativa, como no caso A.P. A natureza e o número das complicações que se podem esperar com esta nova técnica, não devem ser diferentes das encontradas na ventrículo-auriculostomia clássica, por serem relativamente pequenas as modificações introduzidas.

\section{RESUMO}

É relatada uma adaptação da ventrículo-auriculostomia, com válvula de Holter, para casos de bloqueios bilaterais dos orifícios de Monro e de coleções subdurais bilaterais.

A técnica consiste na colocação de dois catéteres de borracha siliconizada nos ventriculos laterais ou espaços subdurais, mediante trepanações paramedianas na região parietal posterior; êstes catéteres são conectados a uma sonda em forma de $\mathrm{T}$ (sonda de Kehr) que, por sua vez, é ligada à uma válvula de Holter, sendo os demais tempos cirúrgicos idênticos aos da ventriculo-auriculostomia convencional.

Foram operados, com esta técnica, três pacientes: dois com craniofaringiomas e um com coleção subdural bilateral. Os resultados foram satisfatórios. 


\section{SUMMARY}

\section{Bilateral ventricular and subdural shunt with the Holter valve: a new surgical technique}

The authors report an adaptation of the ventriculo-auriculostomy, with the Holter valve, for cases of bilateral obstruction of the foramina of Monro and for bilateral subdural fluid collection.

Two catheters of silicone are placed into the lateral ventricles or in the subdural spaces, through para-median burr openings made in the posterior parietal area; these catheters are connected to a T-shaped tube (Kehr tube), which in turn is attached to the end of a Holter valve; the other operative stages are the same as in the conventional ventriculo-auriculostomy.

With this procedure three patients have been operated on: two with craniopharyngioma and one with bilateral subdural fluid collection. The results were good.

\section{REFERENCIAS}

1. ANDERSON, F. M. - Ventriculo-auriculostomy in the treatment of hydrocephalus. J. Neurosurg. 16:551-557, 1959. 2. ARMENISE, B.; DEL VIVO, R. E. \& ISLER, W. - La ventricolo-auriculostomia nel trattamento dell'idrocefalo. Risultati e complicazione in 30 osservazioni di pazienti operati. Minerva neurochirurg. 7:70-72, 1963. 3. BIANCHI, M. \& MIGLIAVACCA, F. - Derivazioni liquorali extratecali. Minerva neurochirurg. 7:57-64, 1963. 4. CASSINARI, V.; MIGLIORI, A. VILLANI, R. - Le traitment de l'hydrocéphalie par drainage extracranien. Observations d'après 113 cas. Neuro-Chirurgie 9:329-337, 1963. 5. ELKINS, C. W. \& FONSECA, J. E. - Ventriculovenous anastomosis in obstructive and acquired communicating hydrocephalus. J. Neurosurg. 18:139-144, 1961. 6. LANGE, M. S. A. Traitment de l'hydrocéphalie par drainage de Spitz-Holter. Neuro-Chirurgie 8:336337, 1962. 7. McNAB, G. M. - The Spitz-Holter valve. J. Neurol. Neurosurg. Psychiat. 22:82-83, 1959. 8. MIGLIORI, A. - Osservazioni sull'impiego della valvola de Spitz-Holter nel trattamento dell'idrocefalo. Minerva neurochirurg. 6:35-40, 1962. 9. NOOONAN, J. A. \& EHMKE, D. A. - Complications of ventriculovenous shunts for control of hydrocephalus. Report of three cases with thromboemboli to the lungs. N. Y. St. J. Med. 269:70-74, 1963. 10. NULSEN, F. E. \& SPITZ, E. B. - Treatment of hydrocephalus by direct shunt from ventricle to jugular vein. Surg. Forum 2: 399-403, 1952. 11. POBLETE, R.; BASAURI, L. \& CHIORINO, R. - Tratamiento de la hidrocefalia con sistemas de derivación del liquido cefalorraquideo al torrente circulatorio. Neurocirurgia 21:35-38, 1963. 12. POPPEN, R. L. - An Atlas of Neurosurgical Techniques. W. B. Saunders Co., Philadelphia, 1960. 13. PUDENZ, R. H.; RUSSELL, F. E.; HURD, A. H. \& SHELDEN, C. H. - Ventriculo-auriculostomy. A technique for shunting cerebrospinal fluid into the rigth auricle. J. Neurosurg. 14:171-179, 1957. 14. SCARF, J. E. - Treatment of hydrocephalus: an historical and critical review of methods and results. J. Neurol. Neurosurg. Psychiat. 26:1-26i, 1963. 15. SPITZ, E. B. - Neurosurgery in the prevention of exogenous mental retardation. Pediat. Clin. N. Amer. 6:1215-1235, 1959. 16. YASHON, D. - Prognosis in infantile hydrocephalus. Past. and present. J. Neurosurg. 20:105-111, 1963.

Clínica Neurológica. Hospital das Clínicas da Faculdade de Medicina da Universidade de São Paulo - Caixa Postal 3461 - São Paulo, Brasil. 